Check for updates

Cite this: Phys. Chem. Chem. Phys., 2022, 24, 5109

Received 18th December 2021, Accepted 31st January 2022

DOI: $10.1039 / \mathrm{d} 1 \mathrm{cp} 05785 \mathrm{e}$

rsc.li/pccp

\title{
Nitrene formation is the first step of the thermal and photochemical decomposition reactions of organic azides $\dagger$
}

\author{
Juan Soto, (D) *a Manuel Algarra (D) ${ }^{b}$ and Daniel Peláez ${ }^{\mathrm{c}}$
}

\begin{abstract}
In this work, the decomposition of a prototypical azide, isopropyl azide, both in the ground and excited states, has been investigated through the use of multiconfigurational CASSCF and MS-CASPT2 electronic structure approaches. Particular emphasis has been placed on the thermal reaction starting at the $\mathrm{S}_{0}$ ground state surface. It has been found that the azide thermally decomposes via a stepwise mechanism, whose rate-determining step is the formation of isopropyl nitrene, which is, in turn, the first step of the global mechanism. After that, the nitrene isomerizes to the corresponding imine derivative. Two routes are possible for such a decomposition: (i) a spin-allowed path involving a transition state; and (ii) a spinforbidden one via a $\mathrm{S}_{0} / \mathrm{T}_{0}$ intersystem crossing. Both intermediates have been determined and characterised. Their associated relative energies have been found to be quite similar, 45.75 and 45.52 $\mathrm{kcal} \mathrm{mol}^{-1}$, respectively. To complete this study, the kinetics of the singlet and triplet channels are modeled with the MESMER (Master Equation Solver for Multi-Energy Well Reactions) code by applying the RRKM and Landau-Zener (with WKB tunnelling correction) theories, respectively. It is found that the canonical rate-coefficients of the singlet path are 2-orders of magnitude higher than the ratecoefficients of the forbidden reaction. In addition, the concerted mechanism has been investigated that would lead to the formation of the imine derivative and nitrogen extrusion in the first step of the decomposition. After a careful analysis of CASSCF calculations with different active spaces and their comparison with single electronic configuration methods (MP2 and B3LYP), the concerted mechanism is discarded.
\end{abstract}

\section{Introduction}

Organic azides $\left(R-N_{3}\right)$ play significant roles in a plethora of fields ranging from chemical synthesis, biological chemistry and pharmaceutics, to technological applications. For example, they are important components in click chemistry ${ }^{1-3}$ or synthesis of energetic materials. ${ }^{4-12}$ Most of these applications as well as their exceptional reactivity arise from their ability to generate nitrene intermediates after $N_{2}$ extrusion. $^{13-17}$ Although organic azides are known for more than a century, ${ }^{18}$ the decomposition reaction mechanism at an atomistic level is

\footnotetext{
${ }^{a}$ Department of Physical Chemistry, Faculty of Science, University of Málaga, E-29071 Málaga, Spain. E-mail: soto@uma.es

${ }^{b}$ INAMAT2 Institute for Advanced Materials and Mathematics, Department of Sciences, Campus de Arrosadia, 31006 Pamplona, Spain

${ }^{c}$ Institut des Sciences Moléculaires d'Orsay (ISMO) - UMR 8214, Université ParisSaclay, 91405 Orsay Cedex, Spain

$\dagger$ Electronic supplementary information (ESI) available: Tables of adiabatic excitation energies, relative energies, and of geometries in internal and cartesian coordinates, details of potential energy surfaces, and input files to MESMER (TAR.GZ). See DOI: 10.1039/d1cp05785e
}

still under discussion. More specifically, there is a lack of agreement in the nature (concerted or asynchronous) of the so-called $\alpha, \beta$-migration reaction in azides (Refs. 19 and 20 to cite both examples). In this process, a group attached to the neighboring $C_{\alpha}$ (for example an $\mathrm{H}$-atom or another Rsubstituent, see Scheme 1) migrates to the $N_{\beta}$. The presence of an intermediate would imply an asynchronous mechanism whereas a lack thereof would mean a concerted one (Scheme 1). To the best of our knowledge, such a controversy arises from two important sources: (i) the theoretical approach used to study the reaction; (ii) the energy barrier for $\alpha, \beta$-migration starting at the nitrene intermediate is usually very low. ${ }^{19-23}$

Hence, the main aim of this work is to shed some light on this issue. To reach this end, we have studied the reaction mechanism and kinetics of the thermal and photochemical decompositions of isopropyl azide $\left[\left(\mathrm{CH}_{3}\right)_{2} \mathrm{CHN}_{3}\right]$. This alkyl azide has been selected for two reasons: (a) the thermolysis of this azide was experimentally studied by Bock and Dammel; ${ }^{24}$ (b) it has a unique migrating hydrogen atom. Unfortunately, to the best of our knowledge, there is no published data on the photochemistry of the title compound. Thus, the only available 

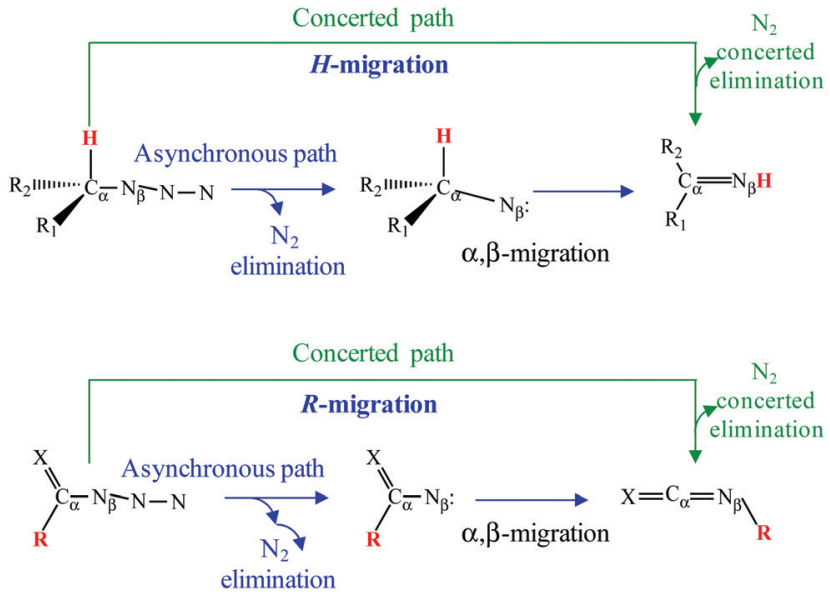

Scheme 1 Proposed reaction mechanisms for decompositions of azides.

values are the calculated parameters (vertical excitations and oscillator strengths) by ourselves corresponding to the photochemical reaction of an analogous alkyl azide. ${ }^{19}$

In this work, we have performed a theoretical study using the complete active space self-consistent (CASSCF) method, which is compared with the data obtained from two commonly used single electronic configuration methods: (1) second order Møller-Plesset theory (MP2) and (2) density functional theory (DFT). Our results will demonstrate that the decomposition proceeds by a stepwise mechanism (asynchronous channel), whose initial step is the extrusion of $\mathrm{N}_{2}$ leading to the formation of the isopropyl nitrene intermediate. Furthermore, it is observed that two channels for nitrene formation exist: (i) via singlet and (ii) via triplet states. In addition, it will be shown through analysis of the kinetics of the singlet and triplet channels, and it is found that the formation of singlet nitrene is the main reaction channel in the decomposition of the studied azide.

\section{Computational details}

Multiconfigurational geometry optimizations of all the relevant species and intermediates of this work have been performed with the CASSCF ${ }^{25-33}$ method in conjunction with the ANO-RCC and ANO-R basis sets, ${ }^{34-36}$ as they are implemented in the MOLCAS 8.4 program. ${ }^{37,38}$ In addition, the energies of such species have been corrected by the multi-state second-order perturbation (MS-CASPT2) ${ }^{39,40}$ method by applying an imaginary shift equal to $0.1 \mathrm{in}$ order to avoid the inclusion of intruder states, and the empirical correction (IPEA) was fixed at 0.25 in all the calculations. State average CASSCF calculations are denoted as SA $n$-CASSCF, where $n$ denotes the number of states of a given symmetry included in the calculation. Spin-orbit coupling constants (SOCC) of intersystem crossing (ISC) have been computed with a spin-orbit Fock-type Hamiltonian. ${ }^{41-43}$

Single electronic configuration calculations have been carried out with the Møller-Plesset (MP2) ${ }^{44}$ and DFT/B3LYP ${ }^{45}$ methods in conjunction with the def2-TZVPPbasis sets. ${ }^{46,47}$
Graphical analysis of the molecular geometries, vibrational normal modes and vectors defining the seam of crossing of minimum energy crossing points (MECP) has been performed with the program MacMolplt. ${ }^{48}$ Analysis of molecular orbitals have been carried out with the programs Gabedit ${ }^{49}$ and Molden. ${ }^{50}$

The labelling convention for the electronic states is based on their energetic ordering $\left(S_{0}, S_{1}, \ldots ; T_{0}, T_{1}, \ldots\right)$, where 0 denotes the lowest energy state in each spin state (singlet, $\mathrm{S}$, or triplet, $\mathrm{T}$ ). The construction of the potential energy curves (PECs) has been done with a linear interpolation method ${ }^{51-55}$ using the full space of nonredundant internal coordinates, which are built as follows. First, a common set of $3 \mathrm{~N}-6$ internal coordinates is defined for the target geometries, the reactants $(R 1)$ and the products $(R 2)$. Note that in the case of dissociation the fragments are separated by a physically reasonable distance $[\Delta(R 2-R 1)]$. Our calculations show that a value of $\sim 4.7 \AA$ for the dissociative bond (in this work, the $N-N_{2}$ ) is enough to reach the asymptotic limit of the potential energy surface (PES) with respect to dissociation of the azide into nitrene and $N_{2}$. This leads to an excellent agreement between the experimental and calculated enthalpies of dissociation. ${ }^{56,57}$ Second, the difference between $R 2$ and $R 1$ yields an interpolation vector $(\Delta R)$ that connects the reactants and products. Third, $\Delta R$ is divided into $n$ segments. This parameter is chosen in order to ensure a smooth convergence of the CASSCF wavefunction upon a geometrical distortion following the $\Delta R$ hyperline. Consequently, each of the divisions constitutes an interpolation step corresponding to a given nuclear conformation on $\Delta R$. The $m$-th one is given by $R_{m}=R 1+(m / n)$ with $m=1, \ldots, n$. Given that our interpolation vectors result from the combination of valence coordinates (internuclear distances, valence bond and dihedral angles), we cannot give a unique unit for them and, in what follows, we will describe them as arbitrary units. Linear interpolations in internal coordinates present two main features that make them particularly appealing to these studies: (i) they are less expensive than scans with relaxation of geometry; (ii) all the points along the interpolation vector (reaction coordinate) are necessarily in a straight line within the set of defined coordinates hence providing a suitable set of coordinates for the representation of reduced dimensionality potential energy surfaces.

Finally, the rate constants associated with transition states (adiabatic reactions) as well as intersystem crossings (nonadiabatic reactions) have been determined with the program MESMER version 5.2. ${ }^{5-62}$ The microcanonical rate constants of transitions states have been computed using the Rice and Ramsperger-Kassel-Marcus (RRKM) theory ${ }^{63-66}$ and the microcanonical rate coefficients of ISCs have been calculated with the Landau-Zener ${ }^{67,68}$ model with inclusion of tunnelling corrections as described by Harvey and Aschi. ${ }^{69}$

\section{Results and discussion}

\section{Selection of the active spaces of isopropyl azide}

Given that we are interested in disentangling whether or not $\alpha, \beta$-migration is involved in the initial step of the decomposition of azides, isopropyl azide has been studied with two active 
spaces of different sizes, including or not the bonding/antibonding pair or molecular orbitals (MOs) describing the $\sigma(\mathrm{CH})$ bond $\left(C_{\alpha}\right.$ in Scheme 1$)$. These two active spaces have been carefully chosen in order to avoid misleading results. ${ }^{70-73}$ This, in turn, has allowed us to calibrate the reliability of our calculations. The largest active space comprises 16 electrons distributed in 14 MOs (Fig. 1). These correspond to: the $2 \mathrm{~s}(\mathrm{~N})$ of the $\mathrm{N}$ atom closest to $C_{\alpha}$, the bonding/antibonding $\sigma(\mathrm{CH})$ pair involving the $C_{\alpha}$, and the migrating $\mathrm{H}$, two bonding/antibonding $\sigma(\mathrm{NNN})$ couples, two bonding/antibonding $\pi(\mathrm{NNN})$ couples and one non-bonding $\pi(\mathrm{NNN})$ orbital. The MOs included in the second smaller active space are the same as before except for the bonding/antibonding $\sigma(\mathrm{CH})$ couple, as a consequence, it comprises 14 electrons and 12 orbitals.

\section{Mechanism of the dissociation of isopropyl azide from the CASSCF multiconfigurational perspective}

In this section, we study the thermal and photochemical dissociation mechanisms of isopropyl azide in the ground and excited states, with special emphasis on the thermal reactions. Unless otherwise specified, all the calculations in this section have been performed under $C_{\mathrm{s}}$ symmetry with the CASSCF method and with an active space of 16 electrons and 14 orbitals. Thus, in a first stage, we have calculated (Table 1) the vertical excitation energies of the low-lying singlet and triplet states of the azide with respect to the minimum of the ground state $\left(\mathrm{S}_{0}\right)$; in addition, Table 1 shows the character of the most relevant electronic configurations and the associated oscillator strengths of the singlet states or spin orbit coupling constants $\left(\mathrm{T}_{n} / \mathrm{S}_{0}: n=0,1\right)$ for the triplet states, respectively. Vertically, the lowest excited state $\left(4.19 \mathrm{eV}: 97 \mathrm{kcal} \mathrm{mol}^{-1}\right)$ corresponds to the

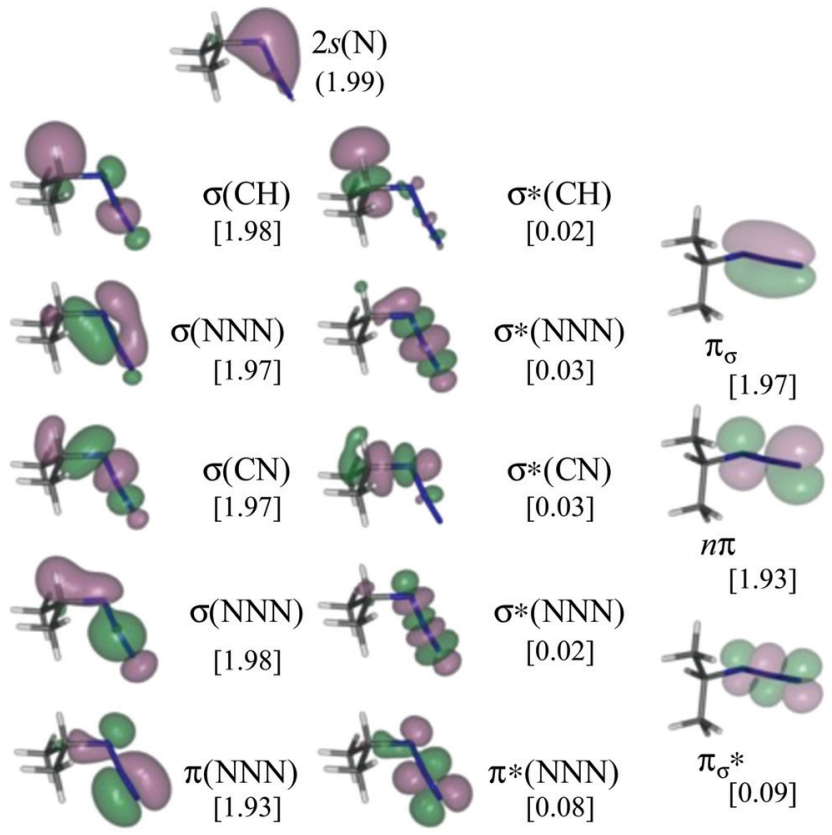

Fig. 1 CASSCF/ANO-RCC natural orbitals included in the active space (16e, 140) of isopropyl azide. In square brackets: occupation numbers of the natural orbitals.
Table 1 Vertical excitation (VE) energies in eV of the singlet and triplet states of isopropyl azide $\left(C_{s}, M S-C A S P T 2\right) .{ }^{a b}$

\begin{tabular}{lllll}
\hline State & VE & $f_{\text {OSC }}{ }^{c}$ & Configuration $^{d}$ & $W^{e}$ \\
\hline $2^{1} \mathrm{~A}^{\prime}$ & 5.87 & $6.4210^{-4}$ & {$[\mathrm{n} \pi]^{1}\left[\pi_{\sigma}^{*}\right]^{1}$} & 39 \\
& & & {$[\pi(\mathrm{NNN})]^{1}\left[\pi^{*}(\mathrm{NNN})\right]^{1}$} & 48 \\
$1^{1} \mathrm{~A}^{\prime \prime}$ & 4.49 & $8.6810-^{-4}$ & {$[\mathrm{n} \pi]^{1}\left[\pi^{*}(\mathrm{NNN})\right]^{1}$} & 89 \\
$2^{1} \mathrm{~A}^{\prime \prime}$ & 7.22 & $5.9410^{-3}$ & {$[\pi(\mathrm{NNN})]^{1}\left[\pi_{\sigma^{*}}\right]^{1}$} & 89 \\
& & SOC $^{f}$ & & \\
$1^{3} \mathrm{~A}^{\prime}$ & 4.66 & 0 & {$[\mathrm{n} \pi]^{1}\left[\pi_{\sigma^{*}}\right]^{1}$} & 75 \\
& & & {$[\pi(\mathrm{NNN})]^{1}\left[\pi^{*}(\mathrm{NNN})\right]^{1}$} & 15 \\
$2^{3} \mathrm{~A}^{\prime}$ & 5.36 & 0 & {$[\mathrm{n} \pi]^{1}\left[\pi_{\sigma^{*}}\right]^{1}$} & 15 \\
& & & {$[\pi(\mathrm{NNN})]^{1}\left[\pi^{*}(\mathrm{NNN})\right]^{1}$} & 73 \\
$1^{3} \mathrm{~A}^{\prime \prime}$ & 4.19 & 16.72 & {$[\mathrm{n} \pi]^{1}\left[\pi^{*}(\mathrm{NNN})\right]^{1}$} & 88 \\
$2^{3} \mathrm{~A}^{\prime \prime}$ & 6.85 & 16.52 & {$[\pi(\mathrm{NNN})]^{1}\left[\pi_{\sigma^{*}}\right]^{1}$} & 89
\end{tabular}

${ }^{a} C_{\mathrm{S}}$ MP2/def2-TZVPP optimized geometry. ${ }^{b} \mathrm{SA2-CASSCF}(16 \mathrm{e}, 140) /$ ANO-RCC reference wave function, IPEA $=0.25$. Imaginary shift $=0.1$. ${ }^{c}$ Oscillator strength. ${ }^{d}$ MS-CASPT2 main electronic configurations of the excited states referred to the ground state configuration. ${ }^{e}$ Weight of the configuration in \%. Only contributions greater than $15 \%$ are included. ${ }^{f}$ Spin orbit coupling constant in $\mathrm{cm}^{-1}$.

first triplet state $\left[\mathrm{T}_{0}: 1^{3} \mathrm{~A}^{\prime \prime}\right]$ and just above it (4.49 eV: $103 \mathrm{kcal}$ $\mathrm{mol}^{-1}$, we have found the first excited singlet state $\left[\mathrm{S}_{1}: 1^{1} \mathrm{~A}^{\prime \prime}\right]$, whose oscillator strength amounts to $\sim 910^{-4}$. Second, in accordance with the linear interpolation method (see above), we have determined the potential energy curves (PECs) for the dissociation processes of the electronic states of Table 1 that would lead to the formation of nitrene species and molecular nitrogen (Fig. 2). Furthermore, these PECs have provided a reasonable guess geometry for subsequent optimization of the critical points related to the sought dissociation channels in the relevant electronic states.

In Fig. 3 and 4, we display the geometries of the relevant critical points. The minimum energy geometry on the ground state $S_{0}\left(M 0: 1^{1} A^{\prime}\right)$ is represented in Fig. 3a. There also exists a minimum energy geometry on the surface of the first excited singlet $\mathrm{S}_{1}\left(\mathrm{M} 1: 1^{1} \mathrm{~A}^{\prime \prime}\right.$ [Fig. 3b]) state which lies $40.7 \mathrm{kcal} \mathrm{mol}^{-1}$

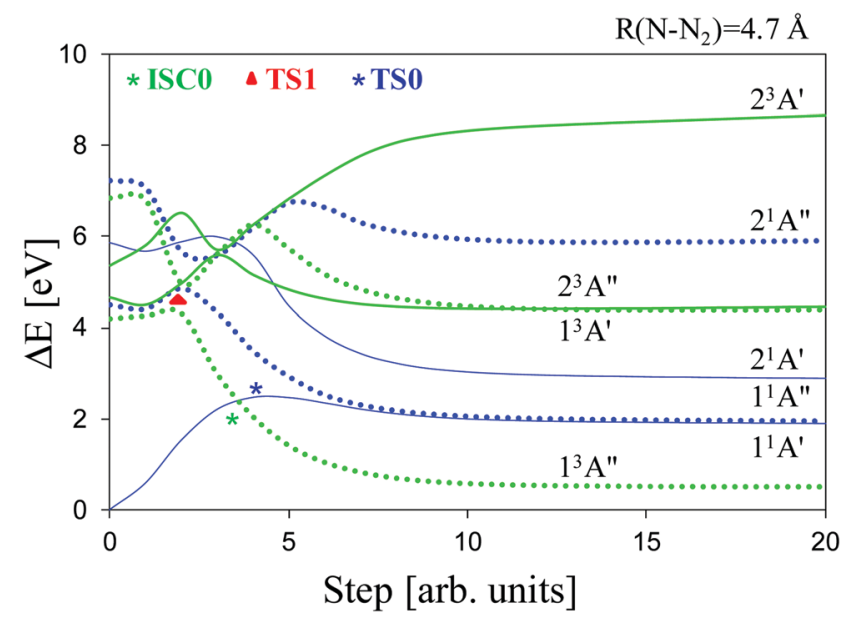

Fig. 2 MS-CASPT2/ANO-RCC potential energy curves of the low-lying singlet and triplet states of isopropyl azide leading to dissociation into isopropyl nitrene and $N_{2}$. Reference wave function: SA2-CASSCF(16e, 140). $A^{\prime}$ singlet states (solid blue lines); $A^{\prime \prime}$ singlet states (blue dotted lines); $A^{\prime}$ triplet states (solid green lines); $A^{\prime \prime}$ triplet states (green dotted lines). 


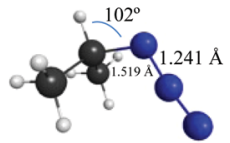

(a) Mo

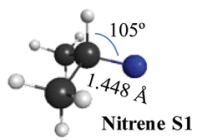

(c)

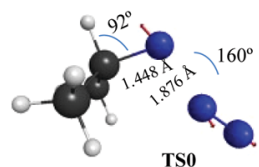

(e)

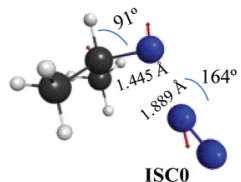

(g)

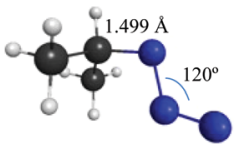

(b) M1

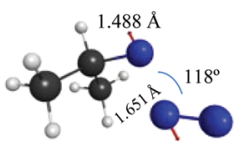

(d) TS1

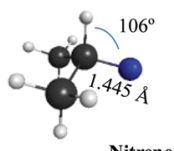

(f)

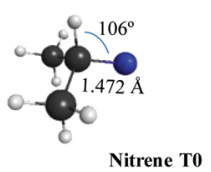

(h)
Fig. 3 CASSCF/ANO-RCC critical points on the potential energy surfaces leading to the dissociation of isopropyl azide into isopropyl nitrene and $\mathrm{N}_{2}$. (a) Minimum azide on $\mathrm{S}_{0}$; (b) minimum azide on $\mathrm{S}_{1}$; (c) minimum nitrene on $\mathrm{S}_{1}$; (d) transition state leading to the dissociation on the $\mathrm{S}_{1}$ surface; (e) transition state leading to the dissociation on the $S_{0}$ surface; (f) nitrene $S_{0}$; (g) intersystem crossing $\mathrm{S}_{0} / \mathrm{T}_{0}$; (f) nitrene $\mathrm{T}_{0}$.

below the $1^{1} \mathrm{~A}^{\prime} \rightarrow 1^{1} \mathrm{~A}^{\prime \prime}$ vertical excitation energy. Concerning the photochemistry of the molecules, the dissociation of the azide into isopropyl nitrene and $\mathrm{N}_{2}$ on the first excited singlet surface $\left(1^{1} \mathrm{~A}^{\prime \prime}\right)$ can occur after surmounting a small barrier to yield singlet nitrene $\left(1^{1} \mathrm{~A}^{\prime \prime}\right.$ [Fig. $\left.\left.3 \mathrm{c}\right]\right)$. The barrier corresponds to a transition state (TS1: $1^{1} \mathrm{~A}^{\prime \prime}$ [Fig. 3d]) that is $2.6 \mathrm{kcal} \mathrm{mol}^{-1}$ above M1 ( $S_{1}$ minimum). In contrast, the first excited triplet state does not present any minimum on the $\mathrm{T}_{0}$ surface around the Franck-Condon region, in fact, this state is dissociative. Concerning the thermal decomposition reaction, two channels for the generation of the nitrene intermediate have been determined: (i) a spin-allowed path, via a transition state on the $\mathrm{S}_{0}$ surface (TS0: $1^{1} \mathrm{~A}^{\prime}$ [Fig. 3e]) $45.8 \mathrm{kcal} \mathrm{mol}^{-1}$ above M0, which yields singlet nitrene $\left(1^{1} \mathrm{~A}^{\prime}\right.$ : [Fig. $\left.\left.3 \mathrm{f}\right]\right)$; (ii) a spin-forbidden path, via an $\mathrm{S}_{0} / \mathrm{T}_{0}$ intersystem crossing process whose MECP lies $45.5 \mathrm{kcal} \mathrm{mol}^{-1}$ above M0 which is represented in Fig. $3 \mathrm{~g}$ (ISC0: $: 1^{1} \mathrm{~A}^{\prime} / 1^{3} \mathrm{~A}^{\prime \prime}$ ) and yields triplet nitrene $\left(1^{3} \mathrm{~A}^{\prime \prime}\right.$ : [Fig. $\left.3 \mathrm{~h}\right]$ ). It is worth noting that the spatial separation between TS0 and ISC0 is only $0.02 \AA$ measured in Cartesian coordinates, a feature that should be important in the dynamics of dissociation through the spin-forbidden channel, given that this fact would imply an enhancement of the probability of intersystem crossing. ${ }^{74}$

The results obtained for the smaller active space are completely analogous to those reported in the previous paragraphs thus evidencing the robustness of our choice of active spaces. Specific data of these calculations are reported in the ESI $\dagger$ (Tables S1 to S8).

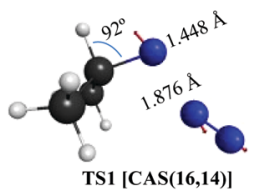

(a)

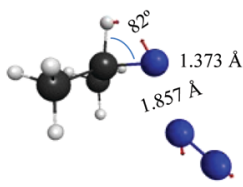

TSx [MP2]

(c)

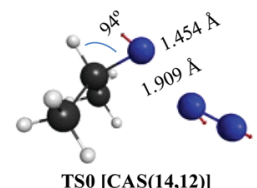

(b)

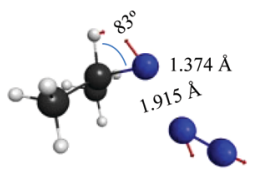

TSx [B3-LYP]

(d)
Fig. 4 Transition states on the potential energy surfaces leading to dissociation of isopropyl azide into isopropyl nitrene and $\mathrm{N}_{2}$ obtained by different methods.

To finish this section, we give the main parameters, energetics and SOCC, calculated with the two basis sets and active spaces applied in this work (Table 2).

\section{Comparison of the decomposition mechanisms obtained from mono- and multiconfigurational methods}

The reaction mechanism of the decomposition of isopropyl azide has been analyzed in the previous section. In here, we compare the CASSCF results with those that are obtained from MP2 and DFT/B3LYP calculations. As was noticed in the Introduction section, there are discrepancies among different theoretical approximations about the mechanism of the reaction (Scheme 1). To be specific, single electronic configuration methods, such as MP2 or DFT/B3LYP, predict a concerted mechanism for the dissociation of organic azides (profiles of the intrinsic reaction coordinates are given in Fig. S1, ESI $\dagger$ ) leading to acetone imine formation as the main product. In contrast, multiconfigurational methods, such as CASSCF, yield an asynchronous mechanism in which isopropyl nitrene is the main intermediate of the first reaction step, but can be formed by two different channels: (i) spin-allowed and (ii) spinforbidden. The geometries of the transition states for the first reaction step obtained with the four different approaches applied in this work are given in Fig. 4. Thus, Fig. 4a and b

Table 2 Energetics (in $\mathrm{kcal} \mathrm{mol}^{-1}$ ) of the dissociation reaction of isopropyl azide into isopropyl nitrene and $\mathrm{N}_{2} \cdot{ }^{a b}$

\begin{tabular}{lllll}
\hline & $\mathrm{BS}^{c}$ & $\mathrm{BS}^{c}$ & $\mathrm{BS}^{e}$ & $\mathrm{BS}^{f}$ \\
\hline$\Delta E(\mathrm{~S})^{g}$ & 45.75 & 46.90 & 47.73 & 49.03 \\
$\Delta E(\mathrm{~T})^{h}$ & 45.52 & 46.39 & 47.34 & 48.46 \\
$\Delta E\left(\mathrm{D}_{\mathrm{S}}\right)^{i}$ & 43.52 & 43.54 & 46.01 & 46.22 \\
$\Delta E\left(\mathrm{D}_{\mathrm{T}}\right)^{j}$ & 11.11 & 11.19 & 13.49 & 13.76 \\
SOCC $^{k}$ & 49.05 & 47.02 & 46.40 & 46.33
\end{tabular}

${ }^{a}$ Calculation referred to the same geometry used in Table $1 .^{b}$ Twostate average wave function. ${ }^{c} \mathrm{CAS}(16,14) / \mathrm{ANO}-\mathrm{RCC} .{ }^{d} \mathrm{CAS}(14,12) / \mathrm{ANO}-$ RCC. ${ }^{e} \mathrm{CAS}(16,14) /$ ANO-R. ${ }^{f} \mathrm{CAS}(14,12) /$ ANO-R. ${ }^{g}$ Energy barrier height of the singlet channel. ${ }^{h}$ Energy barrier height of the triplet channel. ${ }^{i}$ Dissociation energy of the singlet channel. ${ }^{j}$ Dissociation energy of the triplet channel. ${ }^{k}$ Spin orbit coupling constant in $\mathrm{cm}^{-1}$. 
represent the structures calculated with the MP2 and DFT/ B3LYP methods, respectively, and the geometries obtained from the two CASSCF calculations performed with two different active spaces are given in Fig. $4 \mathrm{c}$ d. The arrows in the structures depicted in Fig. 4 correspond to the normal mode of the imaginary frequency associated with the transition vector, which is parallel to the reaction coordinate. From the representation of the transition vectors of Fig. 4, it is clear that the $\alpha-\mathrm{H}$ atom has an important contribution to the reaction coordinate of the MP2 and B3LYP calculations, while the CASSCF results indicate that such an atom does not significantly participate in the dissociation reaction, independent of whether the $\sigma(\mathrm{CH})$ bonding/antibonding molecular orbitals were included or not in the CASSCF calculations. To obtain a quantitative estimation of the participation of each atom in the transition vector for each calculation, after performing the vibrational analysis of these transition states according to the GF method of Wilson, ${ }^{75,76}$ we have computed the potential energy distribution (PED) of the internal coordinates in each normal mode (Table S9, ESI $\dagger$ ). Thus, the values shown in Tables S9 and S10 (ESI $\dagger$ ) corroborate the previous assertion that was based on the pictorial representation of such transition vectors.

\section{Kinetics of the spin-allowed and spin-forbidden channels for isopropyl nitrene formation}

Experimental decomposition of isopropyl azide was studied by Bock and Dammel. ${ }^{24}$ The pyrolysis of the azide was carried out under (nearly) unimolecular conditions $(\sim 1 \mathrm{~Pa})$. It was found that decomposition of the azide began above $600 \mathrm{~K}$ and was completed at $770 \mathrm{~K}$. The main reaction products were the imine derivative and $N_{2}$ In accordance with the experimental conditions, we have computed the coefficient rates for both channels (allowed and forbidden) in the 600-780 K range of temperatures. To achieve this end, we have modeled the kinetics of the dissociation reactions by calculating the reactions rates for the formation of singlet and triplet isopropyl nitrene from the parent azide with the program MESMER. ${ }^{51-55}$ The rate coefficients of the spin-allowed channel have been evaluated with the RRKM theory ${ }^{61-64}$ and the rate coefficients of ISC with the nonadiabatic transition state theory of the Landau-Zener ${ }^{67,68}$ model with inclusion of tunneling corrections. ${ }^{69}$ In Fig. 5, we present the canonical coefficient rates for both channels. The picture obtained from this graphic is crystal clear, and the predominant reaction channel corresponds to the spin-allowed channel whereas the triplet is 2 orders of magnitude lower.

Concerning the calculations of density of states and vibrational partition functions for the MECP geometries, it is interesting to note that, in our calculations, there are two Hessians associated with each MECP (a singlet state Hessian $\mathrm{H}_{1}$ and a triplet state Hessian $\mathrm{H}_{2}$ ). To solve this complication, first, the effective Hessian matrix is introduced which is defined by eqn (1): $:^{77}$

$$
H_{\text {eff }}^{\prime}=\frac{\left|G_{1}\right| H_{2} \pm\left|G_{2}\right| H_{1}}{|\Delta G|}
$$

where $\left|G_{1}\right|$ and $\left|G_{2}\right|$ are the norm of the gradient vectors of the singlet and triplet surface, respectively, and $|\Delta G|$ is the norm of the

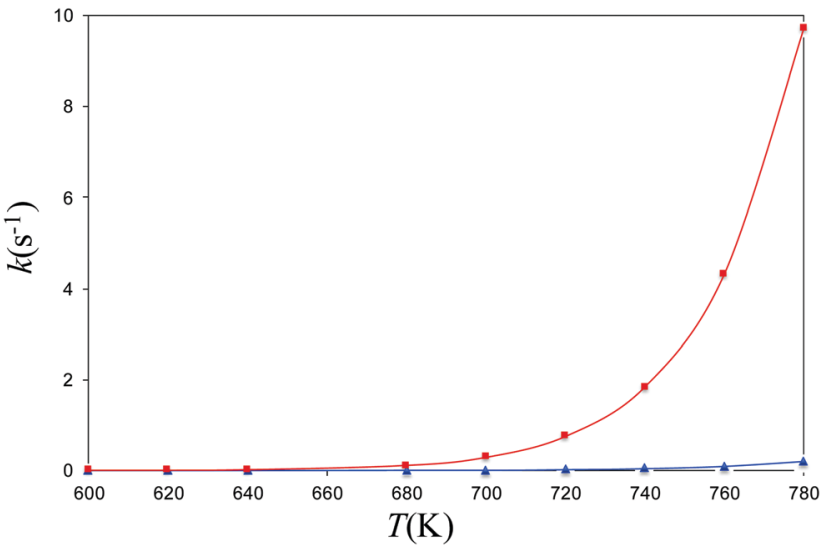

Fig. 5 Canonical rate coefficients as a function of temperature of the singlet and triplet channels for the dissociation of isopropyl azide into isopropyl nitrene and $N_{2}$.

gradient difference vector $\left(G_{1}-G_{2}\right)$. The minus sign is applied if $G_{1}$. $G_{2}>0$ (sloped intersection) and the plus sign when $G_{1} \cdot G_{2}<0$ (peaked intersection).

As usual, $H^{\prime}$ eff has been computed in Cartesian coordinates. This $3 \mathrm{~N} \times 3 \mathrm{~N}$ matrix still contains $3 \mathrm{~N}-6$ vibrational degrees of freedom plus the six rotational-translational degrees of freedom ( $N$ being the number of atoms). Since the structures of the minimum energy crossing points (MECP) are not stationary points on any potential energy surface, the standard vibrational analysis is not valid at such crossing points. To do this properly, it is mandatory to project out from $H^{\prime}$ eff both the rotationaltranslational components as well as the gradient different vector. The projected matrix $H_{\text {eff }}$ is shown in eqn (2). ${ }^{23}$

$$
H_{\mathrm{eff}}=(1-P) H_{\mathrm{eff}}^{\prime}(1-P)^{T}
$$

where $P$ is the projection matrix, which is built as the sum of seven matrices eqn (3)

$$
P=\sum_{j=1}^{7} p_{j}, \text { with } p_{j}=\mathrm{v}_{j} \mathrm{v}_{j}^{T}
$$

where $v_{j}$ (column vector) is the normalized rotational/vibrational mode or the normalized gradient difference vector. It should be noted that the construction of $P$ is greatly simplified by centering the molecule at its center of mass and orientating the principal moments of inertia along the Cartesian axes. The previous operation implies that the same rotation must be applied to $\Delta G$. To finish this section, it must be remarked that, in this work, an initial approach to the calculation of the rate coefficients of the two reaction channels, by taking only the singlet state Hessian in the forbidden process, yielded the opposite answer to the allowed/forbidden ratio, that is, the forbidden path was predicted to be 1-order of magnitude higher than that of the allowed path. 


\section{Conclusions}

We studied the thermal and photochemical decomposition reactions of a prototypical alkyl azide, isopropyl azide, with emphasis on the thermal process. It is found that multiconfigurational methods (CASSCF in our case) are necessary to correctly describe the mechanism. We found that the first and rate-determining step of the reaction is the dissociation of the parent azide into isopropyl nitrene and molecular nitrogen. The prior assertion is applicable for both thermal and photochemical reactions.

The photochemical reaction yields singlet nitrene. On the other hand, the formation of the nitrene intermediate in the thermal decomposition reaction can occur through two different mechanisms: one is a spin-allowed channel that passes through a conventional transition state on the $S_{0}$ ground state surface yielding the singlet nitrene and the other mechanism is a spin-forbidden path which implies $\mathrm{S}_{0} / \mathrm{T}_{0}$ intersystem crossing yielding the triplet nitrene. The modelling of the kinetics of such paths gives the spin-allowed reaction as the major contribution to the formation of nitrene, as a consequence, the nitrene intermediate is formed in its singlet state. Afterwards, the singlet nitrene intermediate can isomerize to the imine derivative through an almost barrierless process. The estimated upper limit for nitrene-imine isomerization is less than 1 kcal mol ${ }^{-1}$ (Fig. S2, ESI $\dagger$ ).

In contrast, the pictures obtained from single electronic methods, such as MP2 and B3LYP, are completely different, that is, both of them give a concerted mechanism in which imine formation is concurrent with elimination of molecular nitrogen.

However, in our opinion, the key point to give an appropriate answer to the problem under study lies in the electronic nature of the nitrene intermediate, which either in its triplet states or in its singlet states must be described by a multiconfigurational reference function. Thus, CASSCF seems to be a most suitable method to deal with the dissociation reaction of azides rather than MP2 or DFT/B3LYP methods.

\section{Conflicts of interest}

The authors declare no conflicts of interest.

\section{Acknowledgements}

This work has been supported by projects UMA18-FEDER-JA049 and P18-RT-4592 of Junta de Andalucía and FEDER founds. The authors thank R. Larrosa, D. Guerrero and F. Moreno for the technical support in running the calculations and the SCBI (Supercomputer and Bioinformatics) of the Univ. Málaga for computer and software resources.

\section{References}

1 A. Dehankar, T. Porter, J. A. Johnson, C. E. Castro and J. O. Winter, J. Chem. Phys., 2019, 151, 144706.
2 M. Danese, M. Bon, G. M. Piccini and D. Passerone, Phys. Chem. Chem. Phys., 2019, 21, 19281-19287.

3 P. J. Silva and C. E. P. Bernardo, J. Phys. Chem. A, 2018, 122, 7497-7507.

4 A. L. Shoaf and C. A. Bayse, New J. Chem., 2019, 43, 15326-15334.

5 S. Kucher, S. Korneev, J. P. Klare, D. Klose and H.-J. Steinhoff, Phys. Chem. Chem. Phys., 2020, 22, 13358-13362.

6 L. Bauer, M. Benz, T. M. Klapötke, T. Lenz and J. Stierstorfer, J. Org. Chem., 2021, 86, 6371-6380.

7 M. V. Gorn, N. P. Gritsan, C. F. Goldsmith and V. G. Kiselev, J. Phys. Chem. A, 2020, 124, 7665-7677.

8 Q. Liang, K. Hayashi, Y. Zeng, J. L. Jiménez-Santiago and D. Song, Chem. Commun., 2021, 57, 6137-6140.

9 M. Schock and S. Bräse, Molecules, 2020, 25, 1009.

10 Q. Li, L. Yan, Z. Wang, Y. Lu, S. Wei, X. Liu and L. Wang, J. Chem. Phys., 2020, 152, 044704.

11 J. Li, M.-K. Liu, Q.-S. Li and Z.-S. Li, Phys. Chem. Chem. Phys., 2020, 22, 28317-28324.

12 J. Y. Park, S. Mondal, H.-J. Kwon, P. K. Sahu, H. Han, K. Kwak and M. Cho, J. Chem. Phys., 2020, 153, 164309.

13 B. A. Shainyan, A. V. Kuzmin and M. Y. Moskalik, Comput. Theor. Chem., 2013, 1006, 52-61.

14 M. Schock and S. Bräse, Molecules, 2020, 25, 1009.

$15 \mathrm{~J}$. Soto and J. C. Otero, J. Phys. Chem. A, 2019, 123, 9053-9060.

16 D. Aranda, F. J. Avila, I. Lopez-Tocon, J. F. Arenas, J. C. Otero and J. Soto, Phys. Chem. Chem. Phys., 2018, 20, 7764-7771.

17 J. Soto, J. C. Otero, F. J. Avila and D. Peláez, Phys. Chem. Chem. Phys., 2019, 21, 2389-2396.

18 T. Curtius, Ber. Dtsch. Chem. Ges., 1890, 23, 3033-3041.

19 M. Algarra, J. Soto, L. Pinto da Silva, M. S. Pino-Gonzalez, J. E. Rodríguez-Borges, J. Mascetti, F. Borget, A. Reisi-Vanani and R. Luque, J. Phys. Chem. A, 2020, 124, 1911-1921.

20 J. F. Arenas, J. I. Marcos, J. C. Otero, A. Sanchez-Galvez and J. Soto, J. Chem. Phys., 1999, 111, 551-561.

21 J. F. Arenas, J. I. Marcos, J. C. Otero, A. Sanchez-Galvez and J. Soto, J. Chem. Phys., 1999, 111, 551-561.

22 M. Algarra and J. Soto, ChemPhysChem, 2020, 21, 1126-1133.

23 J. F. Arenas, J. I. Marcos, I. Lopez-Tocon, J. C. Otero and J. Soto, J. Chem. Phys., 2000, 113, 2282-2289.

24 H. Bock and R. Dammel, J. Am. Chem. Soc., 1988, 110, 5261-5269.

25 B. O. Roos, in Advances in Chemical Physics; Ab initio Methods in Quantum Chemistry II, ed. K. P. Lawley, John Wiley \& Sons, Chichester, UK, 1987, ch. 69, p. 399.

26 B. O. Roos, P. R. Taylor and P. E. M. Siegbahn, Chem. Phys., 1980, 48, 157-173.

27 B. O. Roos, Int. J. Quantum Chem., 1980, 18, 175-189.

28 P. E. M. Siegbahn, J. Almlöf, A. Heiberg and B. O. Roos, J Chem. Phys., 1981, 74, 2384-2396.

29 H.J. Werner and W. Meyer, J. Chem. Phys., 1980, 73, 2342-2356.

30 H.-J. Werner and W. Meyer, J. Chem. Phys., 1981, 74, 5794-5801. 
31 J. Olsen, Int. J. Quantum. Chem., 2011, 111, 3267-3272.

32 D. A. Kreplin, P. J. Knowles and H.-J. Werner, J. Chem. Phys., 2019, 150, 194106.

33 D. A. Kreplin, P. J. Knowles and H.-J. Werner, J. Chem. Phys., 2020, 152, 074102.

34 B. O. Roos, R. Lindh, P.-Å. Malmqvist, V. Veryazov and P.-O. Widmark, J. Phys. Chem. A, 2004, 108, 2851-2858.

35 B. O. Roos, R. Lindh, P.-A. Malmqvist, V. Veryazov and P.-O. Widmark, J. Phys. Chem. A, 2004, 109, 6575-6579.

36 J. P. Zobel, P. O. Widmark and V. Veryazov, J. Chem. Theory Comput., 2020, 16, 278-294.

37 MOLCAS 8.4V. Veryazov, P.-O. Widmark, L. Serrano-Andrés, R. Lindh and B. O. Roos, Int. J. Quantum Chem., 2004, 100, 626-635.

38 F. Aquilante, J. Autschbach, R. K. Carlson, L. F. Chibotaru, M. G. Delcey, L. De Vico, I. Fernández. Galván, N. Ferré, L. M. Frutos, L. Gagliardi, M. Garavelli, A. Giussani, C. E. Hoyer, G. Li Manni, H. Lischka, D. Ma, P. Å. Malmqvist, T. Müller, A. Nenov, M. Olivucci, T. B. Pedersen, D. Peng, F. Plasser, B. Pritchard, M. Reiher, I. Rivalta, I. Schapiro, J. Segarra-Martí, M. Stenrup, D. G. Truhlar, L. Ungur, A. Valentini, S. Vancoillie, V. Veryazov, V. P. Vysotskiy, O. Weingart, F. Zapata and R. Lindh, Molcas 8: New capabilities for multiconfigurational quantum chemical calculations across the periodic table, J. Comput. Chem., 2016, 37, 506-541.

39 B. O. Roos, K. Andersson, M. P. Fülscher, P. Å. Malmqvist, L. Serrano-Andrés, K. Pierloot and M. Merchán, Adv. Chem. Phys., 1996, 93, 219-331.

40 J. Finley, P.-Å. Malmqvist, B. O. Roos and L. Serrano-Andrés, Chem. Phys. Lett., 1998, 288, 299-306.

41 B. A. Heß, C. M. Marian, U. Wahlgren and O. Gropen, Chem. Phys. Lett., 1996, 251, 365-371.

42 P. A. Malmqvist, B. O. Roos and B. Schimmelpfennig, Chem. Phys. Lett., 2002, 357, 230-240.

43 C. Ribbing, B. Gilliams, K. Pierloot, B. O. Roos and G. Karlström, J. Chem. Phys., 1998, 109, 3145-3152.

44 C. Møller and M. S. Plesser, Phys. Rev., 1934, 46, 618-622.

45 A. D. Becke, J. Chem. Phys., 1993, 98, 1372-1377.

46 F. Weigend and R. Ahlrichs, Phys. Chem. Chem. Phys., 2005, 7, 3297-3305.

47 F. Weigend, Phys. Chem. Chem. Phys., 2006, 8, 1057-1065.

48 A. R. Allouche, J. Comput. Chem., 2011, 32, 174-182.

49 G. Schaftenaar and J. H. Noordik, J. Comput.-Aided Mol. Des., 2000, 14, 123-134.

50 B. M. Bode and M. S. Gordon, J. Mol. Graphics Modell., 1998, 16, 133-138.

51 J. Soto, D. Peláez, J. C. Otero, F. J. Avila and J. F. Arenas, Phys. Chem. Chem. Phys., 2009, 11, 2631-2639.
52 D. Pelaez, J. F. Arenas, J. C. Otero and J. Soto, J. Chem. Phys, 2006, 125, 164311.

53 M. Algarra, V. Moreno, J. M. Lazaro-Martinez, E. RodriguezCastellon, J. Soto, J. Morales and A. Benitez, J. Colloid Interface Sci., 2020, 561, 678-686.

54 M. Louleb, L. Latrous, A. Rios, M. Zougagh, E. RodriguezCastellon, M. Algarra and J. Soto, ACS Appl. Nano Mater., 2020, 3, 8004-8011.

55 J. F. Arenas, J. C. Otero, D. Pelaez, J. Soto and L. SerranoAndres, J. Chem. Phys., 2004, 121, 4127-4132.

56 J. Soto and M. Algarra, J. Phys. Chem. A, 2021, 125, 9431-9437.

57 J. Soto, D. Peláez and J. C. Otero, J. Chem. Phys., 2021, 154, 044307. 58 D. R. Glowacki, C. H. Liang, C. Morley, M. J. Pilling and S. H. Robertson, J. Phys. Chem. A, 2012, 116, 9545-9560.

59 P. Pokhilko, R. Shannon, D. Glowacki, H. Wang and A. I. Krylov, J. Phys. Chem. A, 2019, 123, 482-491.

60 L. Onel, R. Lade, J. Mortiboy, M. A. Blitz, P. W. Seakins, D. E. Heard and D. Stone, Phys. Chem. Chem. Phys., 2021, 23, 19415-19423.

61 Z. S. Mir, T. R. Lewis, L. Onel, M. A. Blitz, P. W. Seakins and D. Stone, Phys. Chem. Chem. Phys., 2020, 22, 9448-9459.

62 D. J. Medeiros, S. H. Robertson, M. A. Blitz and P. W. Seakins, J. Phys. Chem. A, 2020, 124, 4015-4024.

63 O. K. Rice and H. C. Ramsperger, J. Am. Chem. Soc., 1927, 49, 1617-1629.

64 (a) L. S. Kassel, J. Phys. Chem., 1928, 32, 225-242; (b) L. S. Kassel, J. Phys. Chem., 1928, 32, 1065-1079.

65 R. A. Marcus and O. K. Rice, J. Phys. Colloid Chem., 1951, 55, 894-908.

66 R. A. Marcus, J. Chem. Phys., 1965, 43, 2658-2661.

67 C. Zener, Proc. R. Soc. London, Ser. A, 1932, 137, 696-702.

68 L. D. Landau, Phys. Z. Sov., 1932, 2, 46-51.

69 J. N. Harvey and M. Aschi, Faraday Discuss., 2003, 124, 129-143.

70 J. Soto, F. J. Avila, J. C. Otero and J. F. Arenas, Phys. Chem. Chem. Phys., 2011, 13, 7230-7231.

71 F. Bernardi, M. Olivucci, M. A. Robb, T. Vreven and J. Soto, J. Org. Chem., 2000, 65, 7847-7857.

72 J. Zhang, J. Peng, D. Hu and Z. Lan, Phys. Chem. Chem. Phys., 2021, 23, 25597-25611.

73 X.-L. Peng, A. Migani, Q.-S. Li, Z.-S. Li and L. Blancafort, Phys. Chem. Chem. Phys., 2018, 20, 1181-1188.

74 D. R. Yarkony, J. Am. Chem. Soc., 1992, 114, 5406-5411.

75 E. B. Wilson, Jr., J. C. Decius and P. C. Cross, Molecular Vibrations, McGraw-Hills, New York, 1955.

76 J. F. Arenas, S. P. Centeno, J. I. Marcos, J. C. Otero and J. Soto, J. Chem. Phys., 2000, 113, 8472-8477.

77 A. O. Lykhin, D. S. Kaliakin, G. E. dePolo, A. A. Kuzubov and S. A. Varganov, Int. J. Quantum Chem., 2016, 116, 750-761. 